\title{
La place des réseaux de correspondants auprès des utilisateurs finaux d'intranet 2.0
}

Le cas d'un établissement bancaire français

Mahdi Azzouz

\section{OpenEdition}

\section{Journals}

Édition électronique

URL : http://journals.openedition.org/communicationorganisation/4367

DOI : 10.4000/communicationorganisation.4367

ISSN : 1775-3546

Éditeur

Presses universitaires de Bordeaux

\section{Édition imprimée}

Date de publication : 1 décembre 2013

Pagination : 135-148

ISBN : 978-2-86781-878-3

ISSN : $1168-5549$

Référence électronique

Mahdi Azzouz, "La place des réseaux de correspondants auprès des utilisateurs finaux d'intranet $2.0 »$, Communication et organisation [En ligne], 44 | 2013, mis en ligne le 01 décembre 2016, consulté le 10 décembre 2020. URL : http://journals.openedition.org/communicationorganisation/4367 ; DOI : https://doi.org/10.4000/communicationorganisation.4367 


\title{
La place des réseaux de correspondants auprès des utilisateurs finaux d'intranet 2.0 \\ le cas d'un établissement bancaire français
}

\author{
Mahdi AZZOUZ'
}

\section{Introduction}

La communication interpersonnelle permet de faire converger des technologies, des activités, voire des acteurs organisationnels éloignés géographiquement. Elle joue un double rôle de collecte et de diffusion des informations (Vaast, 2003). Ce double rôle est considéré comme primordial, notamment dans un contexte de quête de flexibilité (Golden et Powell, 2004). "La flexibilité organisationnelle résulte d'un fort potentiel de communication à la fois interne et externe »(Batra, $2006: 17)$. Ainsi, tout changement escompté résulte généralement de politiques basées sur la communication et pilotées par elle (Ford et Ford, 1995). Plusieurs auteurs en sciences de gestion ont souligné l'importance de la communication dans le processus de flexibilisation de l'organisation (Sushil, 2000 ; Batra, 2006 ; et bien d'autres). Ils considèrent la communication comme étant la base de l'apprentissage organisationnel témoignant des interactions entre les différents agents organisationnels lors de l'usage d'une TSI donnée.

Dans le cas de la technologie-intranet, la communication est un facteur déterminant pour mobiliser et stimuler les ressources organisationnelles. Elle permet à l'organisation de renforcer le succès de son intranet en assurant une meilleure circulation des informations et des documents de travail. La mise en œuvre d'un réseau de correspondants assurant cette communication semble être nécessaire. Ils assurent une proximité par délégation, avec les utilisateurs finaux. Lintérêt de ce réseau est largement accru dans le cas d'une entreprise étendue (réseau distant de bureaux, d'agences ou de représentations). Notre question de recherche est donc la suivante : est-il pertinent de recourir à ce type de dispositif ? Le choix de ce mode d'organisation accélère-t-il l'acceptation de l'intranet 2.0 ? Dans cette optique, nous avons mené une

1 Mahdi AZZOUZ est Docteur en Sciences de Gestion Université de Picardie Jules Verne ; mahdi.azzouz@ voila.fr 
étude de cas au sein d'un établissement bancaire français, nommé ici " $\beta$ " afin de mettre en évidence la place prépondérante des relais de correspondants dans les dispositifs de promotion de l'intranet 2.0. Nous présentons dans un premier temps le cadre conceptuel de la recherche dans lequel nous abordons les effets de ces dispositions sur le résultat d'évaluation de l'intranet. Dans un second temps, nous abordons le cadre empirique de la recherche dans lequel nous explicitons la démarche suivie tout au long de ce travail et les résultats obtenus ainsi que leur discussion.

\section{Présentation du cadre théorique de la recherche}

\section{Intranet : définition et fonctionnalités}

Dans la littérature traitant de l'intranet, un relatif consensus existe pour faire remonter l'apparition de l'intranet au milieu des années 1990. De façon générale, un intranet se fond sur l'usage de la technologie internet appliquée dans le contexte d'une organisation (Tang, 2000). En se référant à l'étude réalisée par Courbon et Tajan (1999:161), «l'intranet désigne la transposition des standards, protocoles et des outils en vigueur dans l'internet au sein des réseaux locaux privés d'entreprises ».

Les différentes applications intranet ont évolué dans les entreprises depuis une vingtaine d'années. Plus récemment, les intranets ont progressé, à l'instar du web, vers une génération 2.0. Le terme web 2.0 (et l'intranet 2.0) désigne une multitude d'innovations d'ordre éditorial, technologique, sociologique qui forment un ensemble, certes disparate, mais qui a changé ou changera, de manière radicale, notre rapport à l'informatique. Le web 2.0 (et l'intranet 2.0) est caractérisé par un certain nombre de traits distinctifs : ergonomie utilisateur plus conviviale, architecture technique plus souple, interopérabilité accrue entre les services et les applications qui le composent, développement des sites collaboratifs dans lesquels les internautes (et les intranautes) sont mis à contribution, et bien d'autres (Balmisse et Ouni, 2009 ; Azzouz, 2012). Portail, gestion de contenu, partage documentaire, travail collaboratif, eServices constituent les outils essentiels de l'intranet 2.0. Afin de synthétiser les multiples usages permis par les intranets, nous retenons trois rôles organisationnels essentiels attribués à ces technologies : informer, communiquer et réaliser des tâches en ligne. Ces trois rôles forment ce que l'on peut appeler intranet informationnel, relationnel et fonctionnel. Chacune de ces fonctions peut être mise en œuvre de façon indépendante des autres.

Cette phase technologique, porteuse de capacités de changements importantes en matière d'usages (Aaron et al. 2010), peut se définir par « un ensemble de méthodes, de technologies, de modèles économiques et sociaux qui se caractérisent par l'ouverture, la participation, des technologies faciles à configurer, et des processus décentralisés » (Alberghini, Cricelti et Grimaldi, $2010: 25)$. 


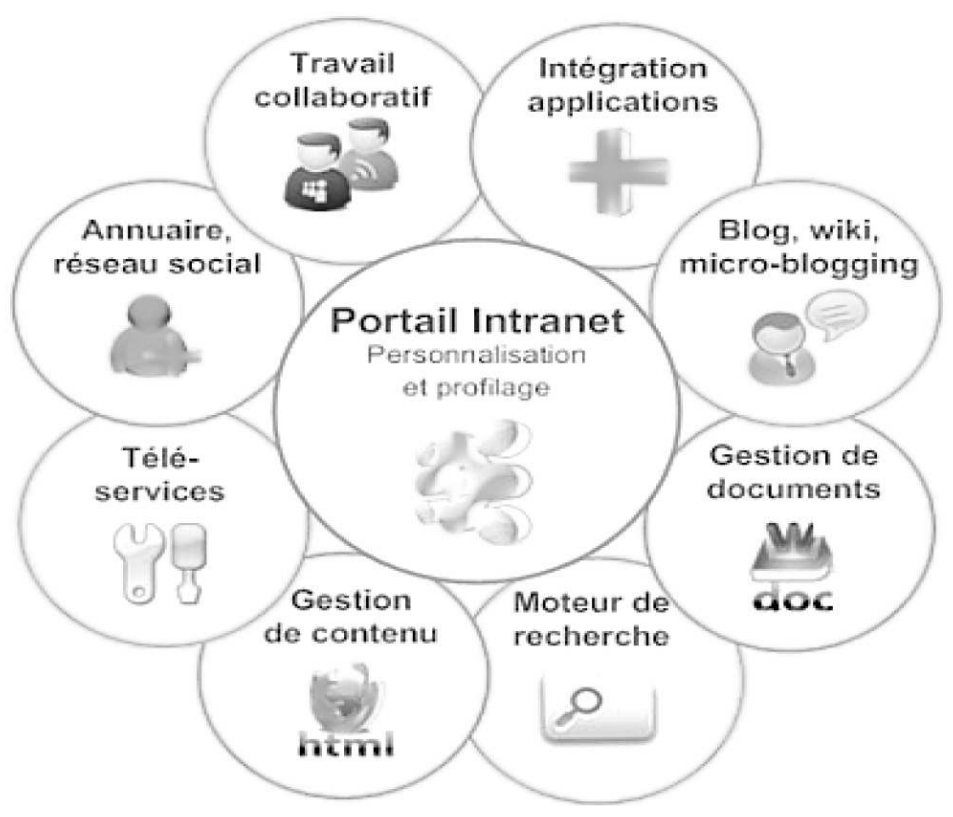

Schéma $\mathrm{n}^{\circ} 1$ : Les fonctionnalités de l'intranet 2.0

\section{Démarche d'évaluation des intranets}

Dans l'ère du "e-management", l'évaluation de la réussite ou du succès des TSI dans les organisations constitue un champ d'étude important pour les sciences de gestion. C'est également une question clé pour les praticiens et les dirigeants des entreprises (Chang et King, 2005). Plusieurs travaux de recherche ont mobilisé le modèle de Delone et McLean (1992, 2003) afin d'étudier le succès des TSI dont des intranets, sous divers angles d'analyse avec des variables dépendantes qui correspondent à l'objet de leur étude. Ce modèle est articulé autour de trois modalités interdépendantes, à savoir : la satisfaction des utilisateurs à l'égard de la technologie, l'usage qui en est fait (autrement dit, leur acceptation du système) et les impacts qui en sont perçus. Dans le cadre de notre travail, nous avons fixé trois critères pour orienter la démarche d'évaluation adaptée à notre terrain de recherche :

- Évaluation post-adoption. Notre évaluation se situe à la phase postadoption de l'intranet, c'est-à-dire une fois que la technologie est adoptée et utilisée de façon assez régulière par les différents acteurs organisationnels. L'intranet est en exploitation au sein de l'établissement bancaire - terrain de recherche - depuis 1997. Il est donc en phase de maturité conformément au cycle d'expérience défini par James, Mulaik et Brett (1982) et repris par un certain nombre d'auteurs en management des TSI (Lee et Kim, 2009 ; Hadoussa et Baile, 2010 ; et bien d'autres); 
- Évaluation multidimensionnelle. Dans le cadre de cette recherche, nous avons adopté une approche multidimensionnelle de la satisfaction, de l'acceptation et des impacts perçus selon laquelle il est possible d'identifier plusieurs dimensions permettant de mesurer chacune de ces trois modalités (Delone et McLean, 1992 ; Deltour, 2004 ; Hadoussa et Baile, 2010 ; Azzouz, 2012). Les chercheurs mettent l'accent sur la nécessité d'adapter le choix des dimensions de l'évaluation en fonction du contexte particulier dans lequel l'organisation se trouve et des différentes perspectives des personnes impliquées dans l'évaluation (Zviran et Erlish, 2003);

- Niveau d'analyse : nous nous sommes focalisé sur le point de vue des utilisateurs finaux pour modéliser un aspect des multiples facettes et fonctions de la technologie - intranet (Deltour, 2004 ; Azzouz, 2012). Les intranautes sont à la fois les producteurs, les organisateurs et les consommateurs de l'information qui circule sur le système d'information organisationnel. Ils sont les mieux placés pour évaluer une technologie mise à leur disposition.

En ce qui concerne la satisfaction des utilisateurs vis-à-vis de l'intranet, huit dimensions ont été retenues, respectivement : l'utilité des contenus de l'intranet, la fiabilité de ces contenus, sa complétude, le design de l'intranet, la simplification d'utilisation de l'intranet, ses qualités techniques, son organisation ; et la facilité de recherche sur l'intranet. Quant à l'acceptation, elle a été appréhendée à travers trois dimensions multidimensionnelles : l'intensité de l'utilisation, l'internalisation et la connaissance de l'intranet. Nous avons retenu également quatre dimensions relatives aux impacts individuels de l'intranet sur les utilisateurs finaux à savoir : l'efficience quantitative de l'intranet, son efficience qualitative, l'intégration organisationnelle de l'intranet et sa connectivité organisationnelle.

\section{Les effets potentiels des dispositifs de promotion sur les résultats d'évaluation}

Dans la littérature en management des TSI, il existe plusieurs facteurs contextuels susceptibles d'influer sur la satisfaction des utilisateurs finaux à l'égard de l'intranet et l'usage qui en est fait (Deltour, 2004 ; Azzouz, 2012). Dans le cadre de ce papier, nous nous penchons uniquement sur les dispositifs de promotion en tant que variable contextuelle au sein du modèle d'évaluation. Les dispositifs de promotion de l'intranet correspondent à l'ensemble des actions de communication mises en place autour de l'intranet dans le but de favoriser sa connaissance auprès des utilisateurs, son utilisation et son acceptation. Ces dispositifs se réfèrent à la publicité de l'intranet par le biais de différents canaux de communication (la diffusion de notes internes, la promotion lors des réunions, la diffusion d'une lettre d'informations, ...) pour mieux informer les utilisateurs sur son contenu, son actualisation, son développement et du rôle de cette technologie dans l'atteinte des objectifs individuels et organisationnels. 
Dans la littérature en management des TSI, peu de recherches ont mobilisé les dispositifs de promotion comme variable contextuelle (Deltour, 2004 ; Azzouz, 2012). Dans notre recherche exploratoire, les dispositifs de promotion ont été évoqués par le responsable maître d'ouvrage intranet et outils collaboratifs, qui précise : «Bien que la mise en place des intranets au sein du groupe date de plusieurs années, l'intranet demeure encore un outil émergent dans les pratiques de travail du personnel. À travers certaines actions de communication (les newsletters, les supports publicitaires, les envois réguliers de messages électroniques portant sur les actualités de l'intranet, ...), nous essayons d'institutionnaliser l'intranet au sein du groupe en encourageant les utilisateurs à le consulter et en les sensibilisant sur l'intérêt de son utilisation régulière pour leur travail quotidien ». Ces dispositifs peuvent favoriser alors l'acceptation de l'intranet par les utilisateurs (Eder et Igbaria, 2001).

Les actions de communication participent également à la constitution d'une perception positive de l'intranet. En renforçant l'image d'un intranet utile, convivial et facile à utiliser, les dispositifs de promotion peuvent influencer favorablement le niveau de satisfaction des utilisateurs à l'égard de l'intranet (Gelderman, 1998).

En s'inspirant des travaux Sushil (2001) et de Sushil et Palanisamy (2003), nous avons retenu quatre items construits autour de trois axes, à savoir : la communication sur les changements induits par l'intranet, sur les bénéfices de cette technologie et sur ses évolutions possibles.

\begin{tabular}{|c|c|c|c|c|c|c|}
\hline \multicolumn{7}{|c|}{ Je suis bien informé(e) des évolutions de contenu de l'intranet $\sim$ CONT2_1 } \\
\hline $\begin{array}{c}\text { Pas du tout } \\
\text { d'accord }\end{array}$ & $\begin{array}{c}\text { Plutôt pas } \\
\text { d'accord }\end{array}$ & $\begin{array}{l}\text { Légèrement } \\
\text { pas d'accord }\end{array}$ & Neutre & $\begin{array}{c}\text { Légèrement } \\
\text { d'accord }\end{array}$ & $\begin{array}{c}\text { Plutôt } \\
\text { d'accord }\end{array}$ & $\begin{array}{c}\text { Tout à fait } \\
\text { d'accord }\end{array}$ \\
\hline \multicolumn{7}{|c|}{ Je suis bien informé(e) des apports de l'intranet pour moi CONT2_2 } \\
\hline $\begin{array}{c}\text { Pas du tout } \\
\text { d'accord }\end{array}$ & $\begin{array}{c}\text { Plutôt pas } \\
\text { d'accord }\end{array}$ & $\begin{array}{l}\text { Légèrement } \\
\text { pas d'accord }\end{array}$ & Neutre & $\begin{array}{c}\text { Légèrement } \\
\text { d'accord }\end{array}$ & $\begin{array}{c}\text { Plutôt } \\
\text { d'accord }\end{array}$ & $\begin{array}{c}\text { Tout à fait } \\
\text { d'accord }\end{array}$ \\
\hline \multicolumn{7}{|c|}{ Les raisons des changements induits par l'intranet me sont suffisamment expliquées CONT2_3 } \\
\hline $\begin{array}{c}\text { Pas du tout } \\
\text { d'accord }\end{array}$ & $\begin{array}{c}\text { Plutôt pas } \\
\text { d'accord }\end{array}$ & $\begin{array}{l}\text { Légèrement } \\
\text { pas d'accord }\end{array}$ & Neutre & $\begin{array}{c}\text { Légèrement } \\
\text { d'accord }\end{array}$ & $\begin{array}{c}\text { Plutôt } \\
\text { d'accord }\end{array}$ & $\begin{array}{c}\text { Tout à fait } \\
\text { d'accord }\end{array}$ \\
\hline \multicolumn{7}{|c|}{$\begin{array}{l}\text { Je trouve que les organes qui s'occupent de l'intranet-groupe communiquent suffisamment sur ses } \\
\text { bénéfices } \sim \text { CONT2_4 }\end{array}$} \\
\hline $\begin{array}{l}\text { Pas du tout } \\
\text { d'accord }\end{array}$ & $\begin{array}{c}\text { Plutôt pas } \\
\text { d'accord }\end{array}$ & $\begin{array}{l}\text { Légèrement } \\
\text { pas d'accord }\end{array}$ & Neutre & $\begin{array}{l}\text { Légèrement } \\
\text { d'accord }\end{array}$ & $\begin{array}{c}\text { Plutôt } \\
\text { d'accord }\end{array}$ & $\begin{array}{l}\text { Tout à fait } \\
\text { d'accord }\end{array}$ \\
\hline
\end{tabular}

Tableau $n^{\circ} 1$ : Echelle de mesure de la perception des dispositifs de promotion - CONT2

\section{Présentation du cadre empirique de la recherche}

\section{Présentation du terrain de recherche}

Les activités des établissements bancaires sont basées en priorité sur l'information et ses traitements. Les études intersectorielles (Rapport annuel du Capgemini, 2008; Observatoire de l'intranet, 2010 ; entre autres) montrent que les banques continuent de jouer un rôle de secteur d'appel pour les TSI. 
Elles sont le lieu d'investissements importants en matière de TSI (Étude réalisée par le cabinet PSB Research, 2008). Les banques sont considérées comme des archétypes d'organisations prospérant grâce à leur maîtrise du traitement de l'information. Le management des TSI constitue donc une clé essentielle de performance dans les activités bancaires.

L'établissement bancaire étudié occupe une place importante sur le marché bancaire français. Il s'agit d'une des principales banques françaises et une des plus anciennes. Elle fait partie des trois piliers de l'industrie bancaire non mutualiste. Le groupe est développé autour de trois grands métiers diversifiés, à savoir : " réseaux de détail, financements spécialisés et assurances ", «banque privée, gestion d'actifs et services aux investisseurs » et «banques de financement et d'investissement ». Le projet intranet a été initié et chapeauté par la direction SI en 1997 puis en collaboration avec la direction RH à partir de l'année 2000. Nous avons identifié quatre structures de gestion de la technologie, à savoir : le comité de pilotage, le comité technique ou opérationnel, le comité d'utilisateur et la cellule en charge de la gestion et de la coordination du SI autour de l'intranet. Le responsable maître d'ouvrage stratégique intranet Groupe et outils collaboratifs joue un rôle essentiel dans ces différents comités. À travers sa fonction, il assure les missions suivantes : " ...organiser le partage d'expériences, coordonner les besoins transverses, organiser la veille et le benchmark et mettre en place des solutions mutualisées " (Responsable maître d'ouvrage intranet et outils collaboratifs). L'intranet contient les principales fonctionnalités traditionnelles (messagerie électronique, forums de discussions, T'chats, ...) s'ajoutant sur les nouveaux services offerts par la génération 2.0 d'intranet (portail groupe, blogs, wikis, flux RSS, tagging, e-room, communautés sociales, ...). L'évolution de l'intranet 1.0 vers une génération 2.0 a commencé à partir de l'année 2005 avec la création des premiers wikis et blogs qui ont connu une croissance significative en 2009 (avec 350 wikis).

\section{Cadre méthodologique}

Notre étude s'inscrit dans le cadre d'une recherche doctorale portant sur l'évaluation de la réussite des intranets : application d'un modèle contextuel d'évaluation multidimensionnelle au sein d'un établissement bancaire français - étude orientée « utilisateur final ». Nous avons adopté une démarche basée sur la triangulation qui consiste à combiner deux approches : qualitative et quantitative, afin de procéder à l'évaluation de l'intranet d'un établissement bancaire français.

- Approche qualitative : dans la phase exploratoire, nous avons privilégié une approche par entretiens, vu la richesse au niveau des informations qu'ils peuvent nous procurer. Ceux-ci nous ont amené à rencontrer les acteurs sur leur lieu de travail afin de rendre compte de la réalité de la situation dans laquelle évoluent ces derniers. Une vingtaine d'interviews ont été recueillies 
au sein d'un groupe bancaire français durant la période 2008-2010. Ils ont été conduits auprès de trois types d'acteurs : les cadres supérieurs, les cadres intermédiaires (ou les managers) et les responsables opérationnels. Ces acteurs sont en prise directe avec l'intranet. Le nom de l'établissement bancaire et des interviewés restent anonymes pour des raisons de confidentialité. Les entretiens sont menés sur la base d'un guide d'entretiens semi-directifs comprenant différents thèmes-questions préalablement élaborés. Les entretiens étaient individuels et de durée variable entre 45 minutes et $1 \mathrm{~h} 30$ chacun. L'ensemble de ces entretiens a été enregistré et retranscrit. Les données issues de ces entretiens ont été encodées et triées selon une technique d'analyse qualitative thématique à travers NVivo 9. L'analyse thématique repose sur la codification du texte par thèmes pour permettre une compréhension en profondeur des représentations et une interprétation synthétique des discours. Nous avons complété cette collecte d'informations par une analyse documentaire. L'ensemble de ces éléments nous a permis, d'une part, de sélectionner les variables faisant partie intégrante de chacune des modalités d'évaluation de l'intranet; et d'autre part, d'élaborer le questionnaire permettant de vérifier la validité et la fiabilité de nos construits (les items).

- Approche quantitative : nous avons opté pour la démarche hypothéticodéductive modérée. Le cadre d'analyse théorique a bénéficié de certains retours issus de notre terrain de recherche. Afin de vérifier la validité et la fiabilité des échelles de mesure retenues au sein du modèle d'évaluation de l'intranet, nous nous sommes appuyés sur deux démarches complémentaires : 1- Le paradigme de Churchill (1979 : 46) qui constitue le processus de validation communément admis en sciences de gestion ; 2- La démarche proposée par Roussel et al. (2002 : 24) qui permet un traitement global du modèle de satisfaction. La taille de notre échantillon $(\mathrm{N}=312)$ satisfait les recommandations méthodologiques pour réaliser les analyses exploratoires et confirmatoires (Kline, 2010). Les différents traitements statistiques effectués dans le cadre de ce travail de recherche sont opérés sous deux logiciels : SPSS (version 17.0) et AMOS (version 19.0).

\section{Résultats et discussions}

Nous présentons les résultats des analyses quantitatives et qualitatives relatives à l'étude des effets des dispositifs de promotion sur la satisfaction des agents organisationnels à l'égard de l'intranet et leur acceptation de cette technologie.

Test des relations entre les trois modalités : satisfaction, acceptation et impacts

Les résultats relatifs à l'ajustement du modèle structurel ci-dessous montrent que l'indice de Chi-deux rapporté au degré de liberté $\left(\chi^{2} / \mathrm{ddl}\right)$ est d'un bon niveau, puisqu'il est de 3,60 $(<5)$. Le RMSEA, qui est inférieur à 0,08 , reflète une très bonne adéquation du modèle théorique global aux 
données empiriques. Quant aux autres indices, plus précisément le GFI, l'AGFI, le NFI et le CFI sont au-dessous des seuils recommandés. Ces indices répondent tous aux normes que nous avons retenues dans notre étude. Nous pouvons dire que le modèle structurel reflète un bon degré d'ajustement aux données collectées et qu'il est donc, à nos yeux, admissible.

\begin{tabular}{|l|l|l|l|l|l|l|l|}
\hline \multicolumn{3}{|c|}{ Indices absolus } & \multicolumn{3}{c|}{$\begin{array}{c}\text { Indices } \\
\text { incrémentaux }\end{array}$} & \multicolumn{3}{c|}{ Indice de parcimonie } \\
\hline GFI & AGFI & RMSEA & CFI & NFI & $\chi^{2}$ & $\mathrm{ddl}$ & $\chi^{2} / \mathrm{ddl}$ \\
\hline 0,94 & 0,89 & 0,063 & 0,938 & 0,912 & 180,16 & 50 & 3,60 \\
\hline$>0,90$ & $>0,80$ & $<0,08$ & $>0,90$ & $>0,90$ & & & $<5$ \\
\hline
\end{tabular}

Tableau $n^{\circ} 2$ : Indices d'ajustement du modèle structurel aux données empiriques

La signification des coefficients entre les variables latentes est étudiée afin d'analyser la structure relationnelle des trois modes d'évaluation de l'intranet.

- L'acceptation est significativement dépendante de la satisfaction des utilisateurs à l'égard de l'intranet $(0,54 ; \mathrm{p}=0,029<0,05)$. La satisfaction des utilisateurs vis-à-vis de l'intranet favorise son acceptation. Plus les utilisateurs sont satisfaits de l'outil, plus ils l'utilisent et plus ils se l'approprient. C'est une relation d'influence positive qui était attendue. L'hypothèse $\mathrm{H} 1$ que nous avons formulée est donc validée. Ce résultat confirme une interrelation relativement forte entre la satisfaction, d'une part, et l'acceptation, d'autre part (Igbaria et Tan, 1997 ; Gelderman, 1998 ; Kim, Suh et Lee, 1998 ; Torkzadeh et Doll, 1999 ; et bien d'autres) ;

- Les impacts perçus sont moyennement influencés par la satisfaction des utilisateurs vis-à-vis de l'intranet $(0,48 ; \mathrm{p}=0,001<0,05)$. Nous soulignons l'existence d'un effet positif mais moyen du niveau de satisfaction des utilisateurs à l'égard de l'intranet sur l'importance des impacts perçus. Les utilisateurs qui expriment plus leur satisfaction pour l'intranet sont ceux qui retirent le plus de bénéfices. L'hypothèse $\mathrm{H} 2$ que nous avons posée est donc partiellement validée. Ce résultat confirme une interrelation relativement forte entre la satisfaction, d'une part, et les impacts perçus d'autre part (Schultz, Ginzberg et Lucas, 1984 ; Delone et McLean, 1992 ; Torkzadeh et Doll, 1999 ; et bien d'autres) ;

- Les impacts perçus sont significativement dépendants de l'acceptation de l'intranet par les utilisateurs finaux $(0,57 ; p=0,001<0,05)$. Il existe un effet positif du niveau d'acceptation de l'intranet par les utilisateurs sur l'importance des impacts perçus. Les utilisateurs les plus réguliers de l'intranet sont ceux qui en retirent le plus de bénéfices. En effet, l'utilisation répétée des différentes fonctionnalités disponibles sur l'intranet dans le travail quotidien des agents organisationnels peut être à l'origine des bénéfices perçus. L'hypothèse $\mathrm{H} 3$ que nous avons formulée est donc validée. Ce résultat confirme une interrelation forte entre l'acceptation, d'une part, et les impacts perçus, d'autre 
part (Goodhue et Thompson, 1995 ; Igbaria et Tan, 1997 ; Gelderman, 1998 ; et bien d'autres). Nous concluons aussi que le niveau des impacts est conditionné, en premier lieu, par le degré d'acceptation et d'incorporation de l'intranet aux pratiques quotidiennes des agents organisationnels, et en second lieu, par le degré de satisfaction de ces agents à l'égard de l'intranet (Igbaria et Tan, 1997 ; Deltour, 2004 ; Masrek, Saad et Ahmad, 2009).

Ces résultats obtenus valident donc les relations théoriques que nous avons posées. Nous avons pu vérifier l'existence d'un enchaînement d'influences allant de la satisfaction vers l'acceptation et les impacts perçus ; et de l'acceptation vers les impacts. Il apparaît clairement le rôle médiateur de l'acceptation qui montre l'importance des comportements d'utilisation dans la perception des effets de l'intranet sur la performance individuelle. L'acceptation constitue donc un indicateur probant du succès des intranets. Dans le cadre de ce papier, nous avons contextualisé ce dispositif d'évaluation afin de renforcer sa pertinence organisationnelle. La variable contextuelle retenue ici correspond aux dispositifs de promotion (CONT2).

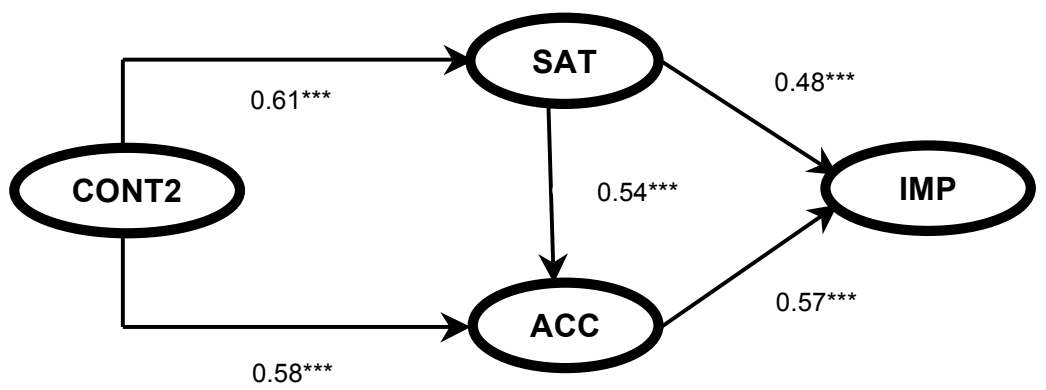

Schéma $n^{\circ} 2$ : Test des effets de l'acculturation à internet et aux technologies mobiles sur l'évaluation des intranets

Effets des dispositifs de promotion sur les résultats d'évaluation

Les résultats obtenus montrent que les dispositifs de promotion (CONT2) agissent positivement aussi bien sur le niveau de satisfaction des utilisateurs à l'égard de l'intranet que sur le degré de leur acceptation de la technologie. Ceci reflète le rôle important de la communication portant sur les changements induits par l'intranet, les bénéfices des changements et les évolutions de ce système. Les deux hypothèses relatives à une influence positive des dispositifs de promotion sur le niveau de satisfaction et le degré d'acceptation sont validées (au seuil $a<5 \%$ ). Nous soulignons donc le rôle important des dispositifs de promotions en tant que leviers d'actions contribuant à faire connaître l'intranet et à faire apprécier le principe de la consultation et de la manipulation d'informations en ligne (Sushil, 2001 ; Sushil et Palanisamy, 
2003). Il est recommandé de créer l'évènement autour de l'intranet (alertes courriels, affichage, teasing ou aguichage, ...).

Un des objectifs que nous avons fixés afin de mettre en pratique les dispositifs de promotion, consiste à créer ou identifier le statut d'animateur au sein des différentes équipes de travail. Qu'il s'agisse d'un dispositif 2.0 utilisant un blog, un wiki ou encore un réseau social, celui-ci repose sur une communauté d'utilisateurs. Ce sont les usages de ces derniers qui déterminent si la mise en place du dispositif est un succès porteur de valeur ou bien d'échec. Dans ce contexte, l'animateur de la communauté revêt un rôle essentiel au niveau local pour dynamiser les intranautes et mettre sous contrôle les éventuels risques de désorganisation et déstabilisation. En tant que volontaire, il va essayer de créer ou soumettre des contenus à la communauté d'utilisateurs, stimuler les échanges interpersonnels, mobiliser les intranautes, dynamiser le développement et la diffusion des nouveaux usages, etc. L'animateur doit également être le garant des règles de bonne conduite généralement matérialisées dans une charte de bonne conduite ${ }^{2}$. Celle-ci doit englober des règles d'or, des bonnes pratiques partagées, entre autres afin d'assurer la cohérence et la cohésion de la communauté d'utilisateurs. Cette charte d'utilisation peut évoluer en fonction des comportements des intranautes. Le but de cette démarche est d'accompagner les salariés ; ce n'est qu'un document juridique.

Le développement de l'intranet et de sa richesse suppose donc l'existence d'un nombre suffisant d'acteurs animateurs qui encadrent les autres utilisateurs et qui assurent une alimentation permanente des contenus. Nous avons proposé dans ce sens de mettre l'accent davantage sur les réseaux de correspondants se situant dans tous les niveaux hiérarchiques de l'établissement bancaire. Ces relais compétents et fusionnant dans l'esprit de l'intranet vont se charger de diffuser la culture intranet au sein des différentes entités organisationnelles et de transmettre les bonnes pratiques associées à l'usage de l'intranet aux autres utilisateurs. Le responsable maître d'ouvrage "intranet et outils collaboratifs" est amené à détecter les acteurs les plus motivés, ceux qui auront fait preuve d'initiatives et seront, à ce titre, susceptibles d'entraîner le groupe dans son sillage.

2 La charte d'utilisation de l'intranet encourage largement les collaborateurs de l'établissement bancaire à utiliser les outils sociaux pour converser avec les autres agents organisationnels. Au-delà de cet encouragement, cette charte poursuit surtout l'objectif d'éviter tout dérapage en ligne en fournissant un certain nombre de conseils comportementaux aux collaborateurs du groupe parmi lesquels : présenter des nouvelles idées créatives sur des sujets internes et externes qui intéressent l'établissement, poster des commentaires respectueux et offrant une réelle valeur ajoutée (pas de spam ou de remarques hors de propos ou provocatrices), répondre rapidement aux commentaires quand une réponse est attendue, garder son sang-froid lorsqu'il y a conflit ou divergence d'opinions avec d'autres intranautes, respecter les droits de propriété intellectuelle et de confidentialité des informations ; et connaître et respecter le code de conduite de l'établissement. 


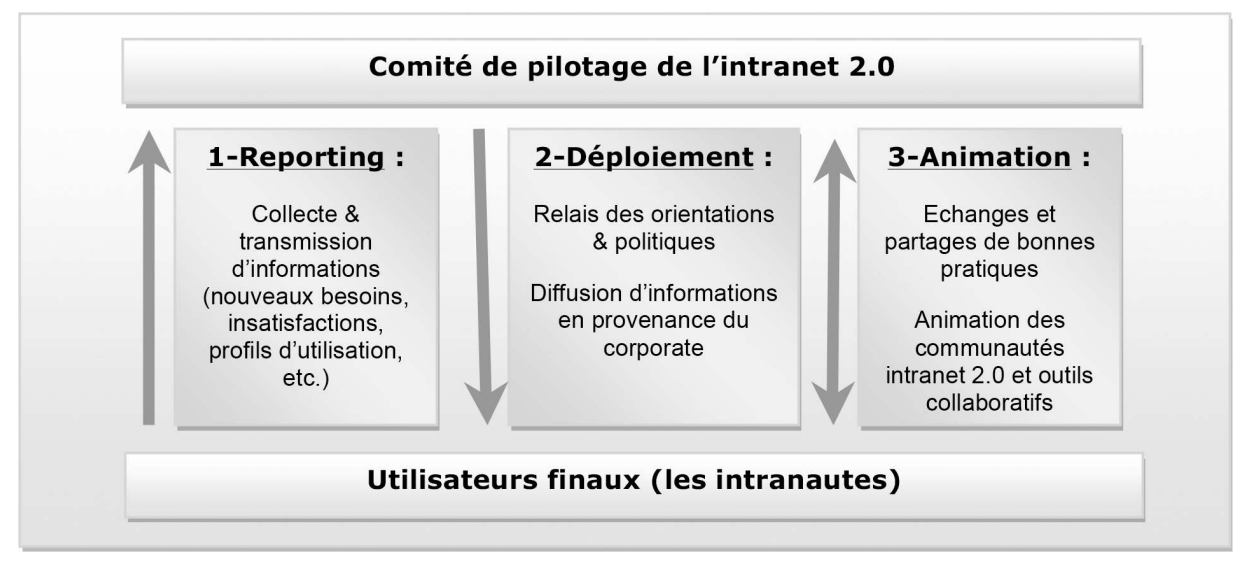

Schéma n`3 : Le rôle du réseau de correspondants dans la promotion de l'intranet 2.0

Cette solution semble être plus réaliste que celle de former l'ensemble des agents organisationnels en matière d'utilisation et de maîtrise de l'outil en cohérence avec l'esprit de l'intranet. Nous rappelons que la mise en place d'un plan de formation de ce genre est particulièrement coûteuse et longue compte tenu du nombre des intranautes appartenant à l'établissement et de leur éloignement géographique.

Certaines entreprises ont tendance à embaucher des stagiaires en tant que chargés de communication interne pour animer leur intranet 2.0. Cette stratégie est assez critiquable : le chargé de communication interne, appelé aussi animateur intranet 2.0, doit disposer de certaines compétences supplémentaires. Autrement dit, il doit avoir des connaissances approfondies sur la culture d'entreprise, son mode de fonctionnement, les relations interpersonnelles, la stratégie de l'entreprise, etc. Nous pensons qu'il est plus approprié de recourir donc au recrutement en interne afin de mieux fonder dans l'esprit de la technologie compte tenu du contexte organisationnel particulier.

\section{Conclusion}

Dans cet article, nous avons fondé notre démarche d'évaluation de l'intranet sur le modèle intégrateur de DeLone et McLean (1992). Les résultats obtenus montrent que l'acceptation est un indicateur probant du succès de l'intranet. Nous avons privilégié une mesure des impacts de l'intranet 2.0 basé sur la perspective des usages. Notre recherche met aussi en évidence le rôle influent des réseaux de correspondants dans le processus d'acceptation de l'intranet 2.0. Le recours à ces réseaux de proximité permet de faciliter l'appropriation de cette technologie. Il apparaît également que les dispositifs de promotion menés par les réseaux de correspondants agissent positivement mais indirectement sur la performance individuelle. Les entreprises qui disposent des réseaux les plus développés sont celles dans lesquelles la démarche intranet 2.0 est la plus 
avancée. Si le comité de pilotage de l'intranet est l'artisan du changement, les relais de correspondants peuvent en être le prolongement opérationnel. La réflexion sur les enjeux de ce mode d'organisation nous semble essentielle. La création d'un réseau de correspondants nécessite plusieurs clarifications portant sur le niveau d'investissements du correspondant (en jour/homme, en énergie pour animer les équipes, ...), la stratégie adoptée par la direction, la culture de l'entreprise, etc. Ce qui permet d'orienter la mise en pratique d'un tel réseau (détermination de sa taille, ses missions, ses ressources, ses relations avec les différentes parties prenantes, entre autres). La création et l'animation des réseaux de correspondants sont un projet qui s'inscrit sur le moyen/long terme.

Le passage des impacts individuels aux impacts organisationnels reste encore problématique. Nous proposons donc de l'effectuer dans le cadre de la perspective interactionniste de la technologie en prenant compte du caractère réflexif entre la TSI, les acteurs, et les caractéristiques organisationnelles. Dans une optique managériale, nous pouvons accorder une attention particulière à l'environnement social des intranautes. Nous faisons référence essentiellement à l'équipe de travail et aux supérieurs hiérarchiques directs. D'autres facteurs contextuels, leviers d'actions, peuvent être mobilisés, parmi lesquels : les dispositifs d'assistance aux utilisateurs en cas de dysfonctionnements du système intranet, les dispositifs de formation proposés aux intranautes et les dispositifs de participation.

\section{BIBLIOGRAPHIE}

AARON F. et al., Débat. " Les usages des systèmes d'information dans les grandes entreprises : une rétrospective », Entreprises et histoires, vol. 3, n 60, 2010, p. 170-184.

ALBERGHINI E., CRICELTI L. and GRIMALDI M., «Implementing Knowledge Management Through IT Opportunities: Definition of a Theoretical Model Based on Tools and Processes Classification ", Proceedings of the European Conference on Intellectual Capital, mars 2010, Rome, Italie.

AZZOUZ M., Évaluation de la réussite des intranets: Application d'un modèle contextuel d'évaluation multidimensionnelle au sein d'un établissement bancaire français -Étude orientée "utilisateur final", Thèse de doctorat en Sciences de Gestion, Université de Picardie Jules Verne, 2012.

BALMISSE G. et OUNI A., Du Web 2.0 à l'entreprise : Usages, Applications et Outils, Hermès, Paris, 2009, 240 p.

BATRA S., "Impact of Information Technology on Organizational Effectiveness: A Conceptual Framework Incorporating Organizational Flexibility ", Global Journal of Flexible Systems Management, vol. 7, n 1-2, 2006, p. 15-25

CHANG J. C. and KING W. R., Measuring the Performance of Information Systems: A Functional Scorecard, Journal of Management Information Systems, vol. 22, $n^{\circ} 1$, Summer, 2005, p. 85-115 
COURBON J.-C. et TAJAN S., Groupware et Intranet : vers le partage des connaissances, Dunod, Paris, 1999.

DELTOUR F., Satisfaction, acceptation, impacts : une analyse multidimensionnelle et contextualisée de l'évaluation individuelle des intranets, Thèse de doctorat en Sciences de Gestion, Université de Lille 1, 2004

DELONE W. and MCLEAN E., «Information Systems Success: The Quest for the Dependant Variable », Information Systems Research, vol. 3, n 1, 1992, p. 60-95.

DELONE W. and MCLEAN E., "The Delone and McLean Model of Information Systems Success: A Ten-Year Update ", Journal of Management Information Systems, Spring, vol. 19, n 4, 2003, p. 9-30.

EDER L.B and IGBARIA M., " Determinants of Intranet Diffusion and Infusion », The International Journal of Management Science, vol. 29, n 1, 2001, p. 233-242.

GELDERMAN M., "The Relation Between User Satisfaction, Usage of Information Systems and Performance ", Information E̋ Management, vol. 34, 1998, p. 11-18.

GOLDEN W. and POWELL P., "Inter-Organizational Information Systems: Systems as enablers of Organizational Flexibility ", Journal of Technology Analysis and Strategic Management, vol. 16, n 3, 2004, p. 299-325.

GOODHUE D. L. and THOMPSON R. L., "Task-technology fit and individual performance », MIS Quarterly, vol. 19, n 2, 1995, p. 213-237.

HADOUSSA S. et BAILE, S., « Les effets de la persuasion, de la satisfaction et des flexibilités perçues par les utilisateurs d'un intranet sur le succès de sa mise en œuvre - un modèle de gouvernance de la TI au sein d'une organisation internationale ", $19^{e}$ congrès AIMS, Luxembourg, 2-4 juin, 2010.

IGBARIA M. and TAN M., "The Consequences of Information Technology Acceptance on Subsequent Individual Performance ", Information E Management, vol. 32, n 3, 1997, p. 113-121.

JAMES L. R., MULAIK S. A., and BRETT J. M., Causal analysis Assumptions, models, and data, Beverly Hills: NCA Sage, 1982.

KLINE R. B., Principles and practice of structural equation Modeling, 3rd ed., New York: Guilford Press, 2010.

LEE A. S. and KIM B. G., « Factors Affecting the Usage of Intranet: A Confirmatory Study », Computer in Human Behaviours Journal, vol. 25, 2009, p. 191-201.

MASREK M. N., SAAD M. S. M. and AHMAD I., "Utilizations and Impacts of Intranet: A Malaysian Case ", MASAUM Journal of Basic and Applied Sciences, vol. 1, $\mathrm{n}^{\circ}$ 1, 2009, p. 52-63.

ROUSSEL P., DURRIEU F., CAMPOY E. et EL AKREMI A., Méthodes d'équations structurelles : Recherche et application en Gestion, Economica, Paris, 2002.

SCHULTZ R., GINZBERG M. and LUCAS H., "A Structural Model of Implementation Management Science Implementation ", Applications of Management Science, Supplement 1 », 1984, p. 55-87

SUSHIL K., "Concept of Systemic Flexibility », Global Journal of Flexible Systems Management, vol. 1, n 1, 2000, p. 77-88. 
SUSHIL K. and R. PALANISAMY, « Measurement and Enablement of Information Systems for Organizational Flexibility: An Empirical Study », Journal of Services Research, vol. 3, n 2, 2003, p. 81-103.

TANG S.-M., "An Impact Factor of Intranet Adoption : An Exploratory and Empirical Research ", The Journal of Systems and Software, vol. 51, 2000, p. 157-173.

TORKZADEH G. and DOLL W. J., «The Development of a Tool for Measuring the Perceived Impact of Information Technology on Work », Omega-The International Journal of Management Science, vol. 27, 1999, pp. 327-339.

VAAST E., La construction des territoires et frontières de l'organisation par les pratiques intranets: Une démarche abductive, Thèse de Doctorat en Sciences de Gestion, École Polytechnique, le 12 mars 2003.

ZVIRAN M. and ERLISH Z., "Measuring IS User Satisfaction: Review and Implications ", Communication of the Association for Information Systems, vol. 12, 2003, pp. 81-103.

Résumé : Cet article s'interroge sur le rôle des réseaux de correspondants dans la promotion de l'intranet 2.0 auprès des utilisateurs finaux. Pour cela, nous étudions l'influence de ces réseaux sur les croyances et les attitudes des salariés. En se référant à l'intranaute comme unité d'analyse, les effets directs et indirects des dispositifs de promotion sont testés sur trois modalités d'évaluation des intranets : la satisfaction qui en est retirée, l'usage qui en est fait et les impacts qui en sont perçus. Ce modèle est testé auprès de 312 intranautes appartenant à un établissement bancaire français. Les données collectées sont traitées en recourant à une analyse exploratoire puis confirmatoire. Les résultats obtenus montrent qu'il existe une influence positive des dispositifs de promotion sur le degré de satisfaction et d'acceptation des intranautes. Quant aux impacts perçus, leur influence n'est qu'indirecte. Les relais de correspondants assurent trois formes de communication complémentaires : la communication sur les changements induits par l'intranet 2.0, sur les bénéfices de ces changements et sur les évolutions à venir.

Mots-clés : Intranet, évaluation, acceptation, satisfaction, communication, dispositifs de promotion, réseau de correspondants, analyse contextuelle.

Abstract: The main purpose of this paper is to examine the role of correspondents' network in the intranet promotion. For this, we study the influence of these networks on beliefs, attitudes and behaviours of intranet end-users. In order to test the direct and indirect effects of promotion measures, we used three evaluation methods: user intranet satisfaction, usage acceptance and the perceived impacts. The proposed model is tested on 312 intranet users belonging to a French bank. To process the data, we used both exploratory and confirmatory analysis. The results reveal a positive infuence of promotion measures on the acceptance and satisfaction degree of the intranet users. About the perceived impacts, their influence is only indirect. The correspondents' network ensures three complementary forms of communication: communication about the changes induced by Intranet 2.0, the benefits of these changes and their future developments.

Keywords: Intranet, evaluation, acceptance, satisfaction, communication, promotion measures, correspondents' network, contextual analysis. 